\title{
A double-ended queue with catastrophes and repairs, and a jump-diffusion approximation*
}

\author{
Antonio Di Crescenzo ${ }^{(1)}$ \\ Virginia Giorno ${ }^{(1)}$ \\ Balasubramanian Krishna Kumar ${ }^{(2)}$ \\ Amelia G. Nobile ${ }^{(1)}$ \\ (1) Dipartimento di Matematica e Informatica, Università di Salerno, \\ Via Ponte don Melillo, I-84084 Fisciano (SA), Italy \\ email: \{adicrescenzo, giorno, nobile\}@unisa.it \\ (2) Department of Mathematics, Anna University, Chennai 600 025, India \\ email: drbkkumar@hotmail.com
}

\begin{abstract}
Consider a system performing a continuous-time random walk on the integers, subject to catastrophes occurring at constant rate, and followed by exponentially-distributed repair times. After any repair the system starts anew from state zero. We study both the transient and steady-state probability laws of the stochastic process that describes the state of the system. We then derive a heavy-traffic approximation to the model that yields a jumpdiffusion process. The latter is equivalent to a Wiener process subject to randomly occurring jumps, whose probability law is obtained. The goodness of the approximation is finally discussed.

Keywords Bilateral birth-death processes - Double-ended queues - Transient probabilities · Catastrophes · Disasters · Repairs · Continued fractions · Jump-diffusion processes · Transition densities
\end{abstract}

AMS 2000 Subject Classifications Primary 60J80; Secondary 60J25

*Paper accepted for publication in Methodology and Computing in Applied Probability

The final publication is available at www.springerlink.com (DOI: 10.1007/s11009-011-9214-2) 


\section{Introduction}

The description of numerous types of systems subject to random evolution can be rendered in a more effective way by including the eventual occurrence of catastrophes. This is well known in the contexts of population dynamics (see, for instance, the contributions by Brockwell 1985, Swift 2000 and 2001, and Gani and Swift, 2006 and 2007). In this research area, Chao and Zheng (2003) considered an immigration birth and death process with total catastrophes and studied its transient as well as equilibrium behavior. Other contributions are due to Economou and Fakinos (2003) and (2008), that extend some previous results on birth-death processes with catastrophes to the more general cases of continuous-time Markov chains with catastrophes resulting from a point process, and of the non-homogeneous Poisson process with total or binomial catastrophes. Various results on a class of stochastic models for systems subject to random regulation via disasters are presented in Stirzaker (2006) and (2007). Also the analysis of queueing systems or birth-death processes subject to catastrophes has attracted the attention of several investigators (see, for instance, Krishna Kumar and Arivudainambi 2000; Krishna Kumar and Pavai Madheswari 2003; Di Crescenzo et al. 2008). A suitable approach adopted by Krinik et al. (2005) to determine the transient probability functions of some classical queueing systems with catastrophes uses dual processes, randomization and lattice path combinatorics, whereas a case of finite birth-death processes with catastrophes was considered in Krinik and Mortensen (2007).

Models based on stochastic processes in the presence of catastrophes have been recently exploited also in another field of mathematical biology, with special reference to the description of the interaction between a myosin head and an actin filament. Precisely, in Buonocore et al. (2009) a continuous-time Markov chain-based model and its continuous approximation have been proposed and studied starting from the $M / M / 1 / K$-catastrophe paradigm.

Along the research line traced by the above mentioned articles, in this paper we consider a different birth-death stochastic model subject to catastrophes. We recall that a bilateral birthdeath process characterized by constant birth and death rates, and subject to jumps to state 0 induced by catastrophes, has been studied recently in Di Crescenzo and Nastro (2004) and in Switkes (2004). Here we aim to study an extension of such a stochastic model, which performs a continuous-time random walk on the integers and, in addition, is subject to the occurrence of catastrophes followed by exponentially-distributed repair times. The state-space of this model thus consists in the whole set of integers complemented by a spurious state, say $F$, which is occupied by the system during the repair times. At the end of any repair the system occupies the state zero and starts afresh. It should be mentioned that the idea of an $M / M / 1$ queueing system subject to catastrophes and followed by random repairs has been recently considered in Krishna Kumar et al. (2007).

It is worth pointing out that the basic stochastic process of our model, namely the bilateral birth-death process with constant birth and death rates, can be viewed for instance as describing the state of a double-ended queue in a taxi-passenger system. Double-ended queues have been studied e.g. in Conolly et al. (2002). Here, denoting by $\{N(t) ; t \geq 0\}$ the state of the considered 
system, $N(t)=n>0$ means that at time $t$ there are $n$ customers in the system, $N(t)=-n<0$ means that at time $t$ there are $n$ taxis in the system, $N(t)=0$ means that at time $t$ the system is empty, and $N(t)=F$ means that at time $t$ the system is in a repair period.

The transient analysis of queueing systems is not always easy to be performed, and it leads very often to non-manageable formulas. In many cases one is forced to study the system under heavy-traffic conditions, in order to obtain at least diffusion or continuous approximations. Also systems subject to catastrophes can be approximated in such a way, the approximating process being usually a jump-diffusion process. This is the case of the contribution given in Di Crescenzo et al. (2003), where an $M / M / 1$ queueing system in the presence of catastrophes is studied and is approximated by a Wiener process subject to randomly occurring jumps. A similar result will be obtained hereafter for the system under investigation.

This is the plan of the paper. In Section 2 we study the preannounced double-ended queue with catastrophes and repairs. By exploting its connection with the system in absence of catastrophes we express the state probabilities of the queue in terms of integrals of Bessel functions. The mean and the variance are also investigated, together with the steady state of the system. Section 3 is devoted to develop the approximating procedure that leads to a jump-diffusion process of the Wiener type. We thus analyze the approximating process, and obtain its transient probability density, mean, variance, and steady-state density. In conclusion, in Section 4 we discuss the goodness of the continuous approximation.

\section{Discrete model with catastrophes and repairs}

We consider a system performing a one-dimensional random walk in continuous time on the whole set of integers. Let $\{N(t) ; t \geq 0\}$ be the position of the system at time $t$. The system moves along the real axis such that $\lambda$ is the rate of moving to the right and $\mu$ is the rate of moving to the left (see Conolly, 1971). This random walk process $\{N(t) ; t \geq 0\}$ is also said to be a bilateral birth-death process, with birth rate $\lambda$ and death rate $\mu$; in any small interval $(t, t+\Delta t), \Delta t>0$, a birth occurs with probability $\lambda \Delta t+o(\Delta t)$; a death occurs with probability $\mu \Delta t+o(\Delta t)$. It is clear that in this interval neither a birth nor death takes place with probability $1-(\lambda+\mu) \Delta t+o(\Delta t)$.

Apart from birth and death processes, catastrophes occur at the system according to a Poisson process with rate $\nu$, i.e., the catastrophe occurs at the system in the small interval $(t, t+\Delta t)$ with probability $\nu \Delta t+o(\Delta t)$. Whenever a catastrophe occurs at the system, the system goes into the failure state $F$, i.e., the bilateral birth-death process is subject to catastrophic failure. The repair times of the failed system are i.i.d. according to an exponential distribution with mean $\eta^{-1}$. After a repair of the system is completed, the system immediately returns to state zero and begins to work again ("on" state).

As mentioned in Section 1, $N(t)$ can be seen as the state of a double-ended queue subject to disasters and repairs. We model the system as a continuous-time Markov process with statespace $S=\{F\} \cup \mathbb{Z}=\{F, 0, \pm 1, \pm 2, \ldots\}$. Let $P_{n}(t)=P[N(t)=n], n=0, \pm 1, \pm 2, \ldots$, be the state probability that the system is in state $n$ at time $t$ when the system is working ("on" state) 
and $q(t)$ be the probability that the system at time $t$ is under repair ("failure" state).

For the system under investigation, the Chapman-Kolmogorov forward differential-difference equations for the system state probabilities $P_{n}(t), n=0, \pm 1, \pm 2, \ldots$, and failure state probability $q(t)$ can be written as

$$
\begin{aligned}
& \frac{\mathrm{d} q(t)}{\mathrm{d} t}=-\eta q(t)+\nu[1-q(t)], \\
& \frac{\mathrm{d} P_{0}(t)}{\mathrm{d} t}=-(\lambda+\mu+\nu) P_{0}(t)+\lambda P_{-1}(t)+\mu P_{1}(t)+\eta q(t), \\
& \frac{\mathrm{d} P_{n}(t)}{\mathrm{d} t}=-(\lambda+\mu+\nu) P_{n}(t)+\lambda P_{n-1}(t)+\mu P_{n+1}(t), \quad n= \pm 1, \pm 2, \pm 3, \ldots .
\end{aligned}
$$

Without loss of generality, assume that initial position of the system at time $t=0$ is zero, i.e.,

$$
q(0)=0 \quad \text { and } \quad P_{n}(0)= \begin{cases}1 & \text { if } n=0 \\ 0 & \text { otherwise }\end{cases}
$$

\subsection{Transient distribution and relation with the process in absence of catas- trophes}

Let us now introduce the so-called randomized random walk $\{\tilde{N}(t) ; t \geq 0\}$ with parameters $\lambda$ and $\mu$. It is a bilateral birth-death process over $\mathbb{Z}$ characterized by birth rate $\lambda$ and death rate $\mu$, and describes the state of the system under consideration in the absence of catastrophes. We recall that the state probabilities of process $\tilde{N}(t)$ conditional on $\widetilde{N}(0)=0$ are given by (see, for instance, Baccelli and Massey 1989 or Conolly 1971)

$$
\widetilde{P}_{n}(t)=\mathrm{e}^{-(\lambda+\mu) t} \beta^{n} I_{n}(\alpha t), \quad t \geq 0, \quad n \in \mathbb{Z},
$$

where

$$
\alpha=2 \sqrt{\lambda \mu}, \quad \beta=\sqrt{\frac{\lambda}{\mu}}
$$

and $I_{n}(\cdot)$ is the modified Bessel function of the first kind of order $n$. We remark that the distribution given in (2.5) is often named Skellam distribution.

Hereafter we express the state probabilities $P_{n}(t)$ in terms of suitable quantities concerning the bilateral birth-death process in absence of catastrophes $\tilde{N}(t)$. Indeed, from probabilistic arguments it is not hard to see that the probabilities $P_{n}(t)$ can be expressed in terms of (2.5) as follows:

$$
P_{n}(t)=e^{-\nu t} \widetilde{P}_{n}(t)+\eta \int_{0}^{t} q(\tau) e^{-\nu(t-\tau)} \widetilde{P}_{n}(t-\tau) \mathrm{d} \tau, \quad t \geq 0, \quad n \in \mathbb{Z} .
$$

The first term in the right-hand-side of (2.7) is the probability that the system goes from 0 to $n$ without occurrence of catastrophes in $(0, t)$, whereas the second term represents the probability that at time $\tau \in(0, t)$ the system enters state 0 due to a repair and then goes from 0 to $n$ without occurrence of catastrophes in $(\tau, t)$.

Theorem 2.1 For all $t \geq 0$ we have

$$
q(t)=\frac{\nu}{\eta+\nu}\left[1-e^{-(\eta+\nu) t}\right],
$$




$$
\begin{aligned}
P_{n}(t)= & \beta^{n}\left\{e^{-(\lambda+\mu+\nu) t} I_{n}(\alpha t)+\frac{\eta \nu}{\eta+\nu}\left[\int_{0}^{t} e^{-(\lambda+\mu+\nu) u} I_{n}(\alpha u) \mathrm{d} u\right.\right. \\
& \left.\left.-e^{-\nu t} \int_{0}^{t} e^{-\eta \tau-(\lambda+\mu)(t-\tau)} I_{n}(\alpha(t-\tau)) \mathrm{d} \tau\right]\right\}, \quad n \in \mathbb{Z},
\end{aligned}
$$

where $\alpha$ and $\beta$ are defined in (2.6).

Proof. Making use of Eqs. (2.1) and recalling the first of (2.4) we obtain (2.8). Furthermore, substituting (2.5) and (2.8) in Eq. (2.7) the transient distribution (2.9) follows.

Some plots of probabilities (2.9) are shown in Figure 1. The expressions given in Theorem 2.1 completely determine all state transient probabilities of the system and the failure state probability $q(t)$ for the bilateral birth-death process $N(t)$ with catastrophes and repairs.

Let us now denote by $\widetilde{T}_{0, n}$ the first-passage time from state 0 to state $n(n= \pm 1, \pm 2, \pm 3, \ldots)$ of process $\tilde{N}(t)$, and by $\widetilde{g}_{0, n}(t)$ the corresponding probability density function. It is well known that (see Abate et al. 1991, for instance), for all $t>0$

$$
\widetilde{g}_{0, n}(t)=\frac{|n|}{t} \widetilde{P}_{n}(t)=\frac{|n|}{t} e^{-(\lambda+\mu) t} \beta^{n} I_{n}(\alpha t), \quad n= \pm 1, \pm 2, \pm 3, \ldots
$$

Due to the Markov property, the following renewal-type equation holds for the system under consideration, for $t>0$ :

$$
P_{n}(t)=\int_{0}^{t} P_{0}(u) e^{-\nu(t-u)} \widetilde{g}_{0, n}(t-u) \mathrm{d} u, \quad n= \pm 1, \pm 2, \pm 3, \ldots .
$$

Hence, Eq. (2.11) expresses that any sample-path of $N(t)$ going from 0 to state $n$, at an eventual time $u \in(0, t)$ visits the state 0 and then goes from 0 to $n$ without occurrence of catastrophes in $(u, t)$. Hereafter we provide an alternative expression of the transition probabilities.

Proposition 2.1 For all $t \geq 0$ the following relation holds:

$$
P_{n}(t)=|n| \beta^{n} \int_{0}^{t} e^{-(\lambda+\mu+\nu)(t-u)} \frac{I_{n}(\alpha(t-u))}{t-u} P_{0}(u) \mathrm{d} u, \quad n= \pm 1, \pm 2, \pm 3, \ldots
$$
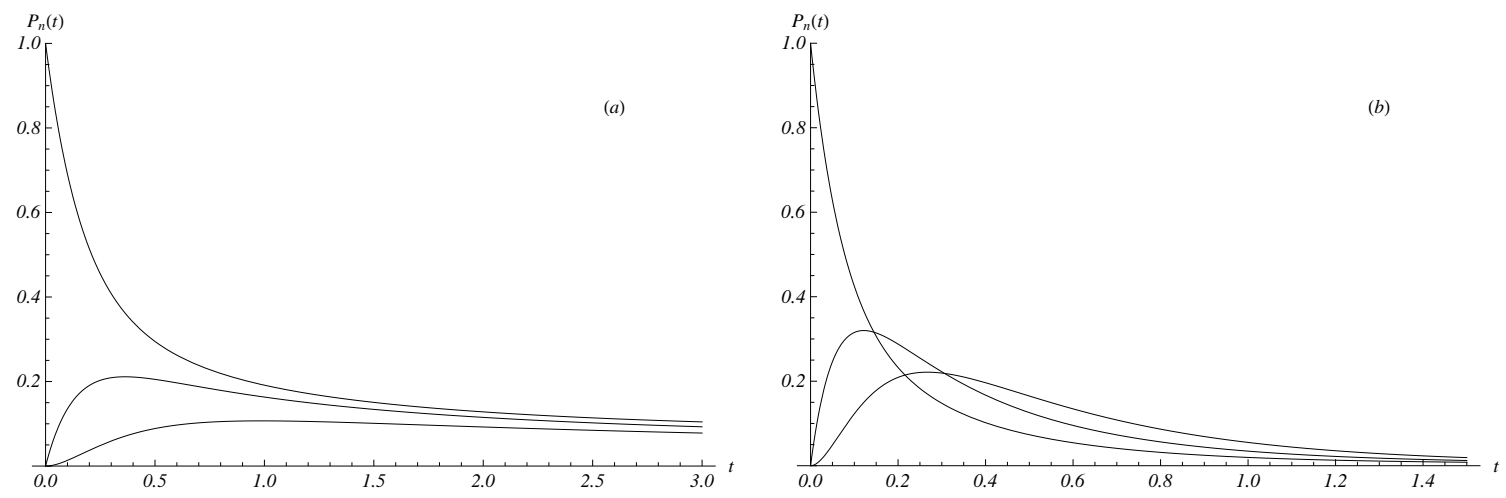

Figure 1: Plots of $P_{n}(t)$ for $n=0,1,2$ (from top to bottom near the origin), where $\mu=2$, $\nu=0.1, \eta=1$, and (a) $\lambda=2$, (b) $\lambda=8$. 
Proof. It immediately follows by making use of (2.10) in (2.11).

In the following, for any function $f(t), t \geq 0$, we denote by $f^{*}(z)=\int_{0}^{\infty} e^{-z t} f(t) \mathrm{d} t, z>0$, its Laplace transform.

By adopting customary methodologies, such as those described in Jain et al. (2007) or Parthasarathy and Lenin (2004), we can evaluate the Laplace transform of the transition probabilities $P_{n}(t)$. Indeed, by taking Laplace transforms in the system of equations (2.1)-(2.3), after some calculations we obtain:

$$
P_{0}^{*}(z)=\frac{1}{\sqrt{(z+\lambda+\mu+\nu)^{2}-4 \lambda \mu}}+\frac{\eta \nu}{z(z+\eta+\nu) \sqrt{(z+\lambda+\mu+\nu)^{2}-4 \lambda \mu}}
$$

and

$$
P_{n}^{*}(z)= \begin{cases}P_{0}^{*}(z)\left[\psi_{2}(z)\right]^{n}, & n=1,2,3, \ldots \\ P_{0}^{*}(z)\left[\psi_{1}(z)\right]^{n}, & n=-1,-2,-3, \ldots\end{cases}
$$

where

$$
\psi_{1}(z), \psi_{2}(z)=\frac{z+\lambda+\mu+\nu \pm \sqrt{(z+\lambda+\mu+\nu)^{2}-4 \lambda \mu}}{2 \mu}, \quad \psi_{1}(z)>\psi_{2}(z)
$$

are the roots of quadratic equation $\mu \chi^{2}(z)-(z+\lambda+\mu+\nu) \chi(z)+\lambda=0$. Expressions (2.12) and (2.13) will be used in Section 4 in order to discuss the goodness of the approximation of $N(t)$ by a suitable jump-diffusion process $X(t)$.

\subsection{Steady-state distribution}

In this section, we shall investigate the structure of the steady-state probabilities and the failure state probability of process $N(t)$ :

$$
\pi_{n}:=\lim _{t \rightarrow \infty} P_{n}(t), \quad q:=\lim _{t \rightarrow \infty} q(t)
$$

Theorem 2.2 The steady-state probabilities of the system state $\left\{\pi_{n}: n=0, \pm 1, \pm 2, \ldots\right\}$ and the failure probability $q$ of the bilateral birth-death process with catastrophes are obtained as, for $\nu>0$ and $\eta>0$,

$$
\begin{aligned}
& q=\frac{\nu}{\eta+\nu}, \\
& \pi_{0}=(1-q) \frac{\nu}{\sqrt{(\lambda+\mu+\nu)^{2}-4 \lambda \mu}}, \\
& \pi_{n}=\left[\frac{(\lambda+\mu+\nu)-\sqrt{(\lambda+\mu+\nu)^{2}-4 \lambda \mu}}{2 \mu}\right]^{n} \pi_{0}, \quad n=1,2, \ldots
\end{aligned}
$$

and

$$
\pi_{-n}=\left[\frac{(\lambda+\mu+\nu)-\sqrt{(\lambda+\mu+\nu)^{2}-4 \lambda \mu}}{2 \lambda}\right]^{n} \pi_{0} \quad n=1,2, \ldots
$$


Proof. The steady-state probabilities of the system can be obtained by letting $t \rightarrow+\infty$ in the system of equations (2.1)-(2.3). The resulting balance equations yield a system of recurrence relations of degree 2 with known coefficients, whose solution can be obtained by standard methods. Eqs. (2.14)-(2.17) then follow.

\subsection{Moments}

Let us now focus on the mean of the bilateral birth-death process with catastrophes and repairs, and set

$$
m_{N}(t)=E\left[N(t) \cdot \mathbf{1}_{\{N(t) \neq F\}} \mid N(0)=0\right], \quad t \geq 0 .
$$

Then, from (2.7) we have

$$
m_{N}(t)=e^{-\nu t} \widetilde{m}(t)+\eta \int_{0}^{t} q(\tau) e^{-\nu(t-\tau)} m_{\widetilde{N}}(t-\tau) \mathrm{d} \tau, \quad t \geq 0
$$

where $m_{\widetilde{N}}(t):=E[\tilde{N}(t) \mid \tilde{N}(0)=0]=(\lambda-\mu) t$ is the mean of the randomized random walk with initial state 0 . Hence, recalling (2.8) after some calculations we obtain

$$
m_{N}(t)=\frac{(\lambda-\mu) \eta}{(\eta+\nu) \nu}\left\{1-e^{-\nu t}+\frac{\nu^{2}}{\eta^{2}} e^{-\nu t}\left(1-e^{-\eta t}\right)\right\} .
$$

Due to (2.18), if $\lambda>\mu$ the following holds:

- if $\eta \geq \nu$ then $m_{N}(t)$ is increasing for all $t>0$,

- if $\eta<\nu$ then $m_{N}(t)$ is increasing for $t<\bar{t}$ and is decreasing for $t>\bar{t}$, where

$$
\bar{t}:=\frac{1}{\eta} \log \frac{\nu}{\nu-\eta}
$$

Clearly, if $\lambda<\mu$ the monotonicity of $m_{N}(t)$ is reversed. Some plots of $m_{N}(t)$ are shown in Figure 2 .

Let us now introduce the variance of the bilateral birth-death process with catastrophes and repairs:

$$
V_{N}(t)=\operatorname{Var}\left[N(t) \cdot \mathbf{1}_{\{N(t) \neq F\}} \mid N(0)=0\right], \quad t \geq 0 .
$$

By adopting a similar procedure, making use of (2.7), (2.18) and recalling that $\operatorname{Var}[\tilde{N}(t) \mid \widetilde{N}(0)=$ $0]=(\lambda+\mu) t$, it is not hard to obtain $V_{N}(t)$. Some plots of the variance of $N(t)$ are given in Figure 3 .

We now discuss the asymptotic mean and variance of process $N(t)$. From (2.18) it is not hard to see that, for $v>0$,

$$
\lim _{t \rightarrow+\infty} m_{N}(t)=\frac{(\lambda-\mu) \eta}{(\eta+\nu) \nu}=(1-q) \frac{\lambda-\mu}{\nu}
$$

We point out that the presence of catastrophes and repairs may be seen as a regulatory effect to the system. Indeed, if $\nu=0$ the system does not admit a steady-state distribution, whereas if $\nu>0$ then the right-hand-side of (2.19) is finite. Finally, straightforward calculations lead to the following asymptotic variance, for $v>0$ :

$$
\lim _{t \rightarrow+\infty} V_{N}(t)=\frac{(\lambda+\mu) \eta}{(\eta+\nu) \nu}+\frac{(\lambda-\mu)^{2} \eta}{(\eta+\nu)^{2} \nu^{2}}(2 \nu+\eta) .
$$




\section{Jump-diffusion approximation}

Let us now construct a continuous approximation starting from the process $N(t)$. We shall make use of a scaling similar to one succesfully used elsewhere in queueing theory contexts (see Giorno et al. 1986, 1987; Di Crescenzo and Nobile 1995). We recall that a popular paper dealing with Brownian motion with jumps that approximates a standard single-server queue with random vacations is due to Kella and Whitt (1990), whereas Kimura (2004) proposed an useful review of some basic concepts and issues in diffusion modeling, and a bibliographical guide to diffusion models for queues that are typically found in computer/communication systems.

Let us consider the Markov process $\left\{N_{\varepsilon}^{a}(t) ; t \geq 0\right\}$, having state space $\{F\} \cup\{\ldots,-\varepsilon, 0, \varepsilon, \ldots\}$, with $\varepsilon>0$. For all $t>0$, the transitions of $N_{\varepsilon}^{a}(t)$ are governed by the following rates:

$$
\begin{array}{ll}
\lim _{\Delta t \downarrow 0} \frac{1}{\Delta t} P\left\{N_{\varepsilon}^{a}(t+\Delta t)=(n+1) \varepsilon \mid N_{\varepsilon}^{a}(t)=n \varepsilon\right\}=\frac{\widehat{\lambda}}{\varepsilon}+\frac{\sigma^{2}}{2 \varepsilon^{2}}, & n \in \mathbb{Z}, \\
\lim _{\Delta t \downarrow 0} \frac{1}{\Delta t} P\left\{N_{\varepsilon}^{a}(t+\Delta t)=(n-1) \varepsilon \mid N_{\varepsilon}^{a}(t)=n \varepsilon\right\}=\frac{\widehat{\mu}}{\varepsilon}+\frac{\sigma^{2}}{2 \varepsilon^{2}}, & n \in \mathbb{Z},
\end{array}
$$

where $\widehat{\lambda}, \widehat{\mu}$ and $\sigma$ are positive parameters. Moreover, the process $\left\{N_{\varepsilon}^{a}(t) ; t \geq 0\right\}$ is still subject to catastrophes arriving with rate $\nu$. Repairs are still exponentially distributed with rate $\eta$, and the system enters state 0 just after a repair. Hence, the repair and failure time distributions are not affected by the scaling procedure introduced above, so that

$$
\begin{array}{ll}
\lim _{\Delta t \downarrow 0} \frac{1}{\Delta t} P\left\{N_{\varepsilon}^{a}(t+\Delta t)=F \mid N_{\varepsilon}^{a}(t)=n \varepsilon\right\}=\nu, & n \in \mathbb{Z}, \\
\lim _{\Delta t \downarrow 0} \frac{1}{\Delta t} P\left\{N_{\varepsilon}^{a}(t+\Delta t)=0 \mid N_{\varepsilon}^{a}(t)=F\right\}=\eta, & n \in \mathbb{Z},
\end{array}
$$

where $\eta$ and $\nu$ are the same parameters of $N(t)$. Essentially, the integers of the state-space of $N(t)$ have been rescaled by a factor $\varepsilon$, and the following substitutions have been performed:

$$
\lambda=\frac{\widehat{\lambda}}{\varepsilon}+\frac{\sigma^{2}}{2 \varepsilon^{2}}, \quad \mu=\frac{\widehat{\mu}}{\varepsilon}+\frac{\sigma^{2}}{2 \varepsilon^{2}}
$$
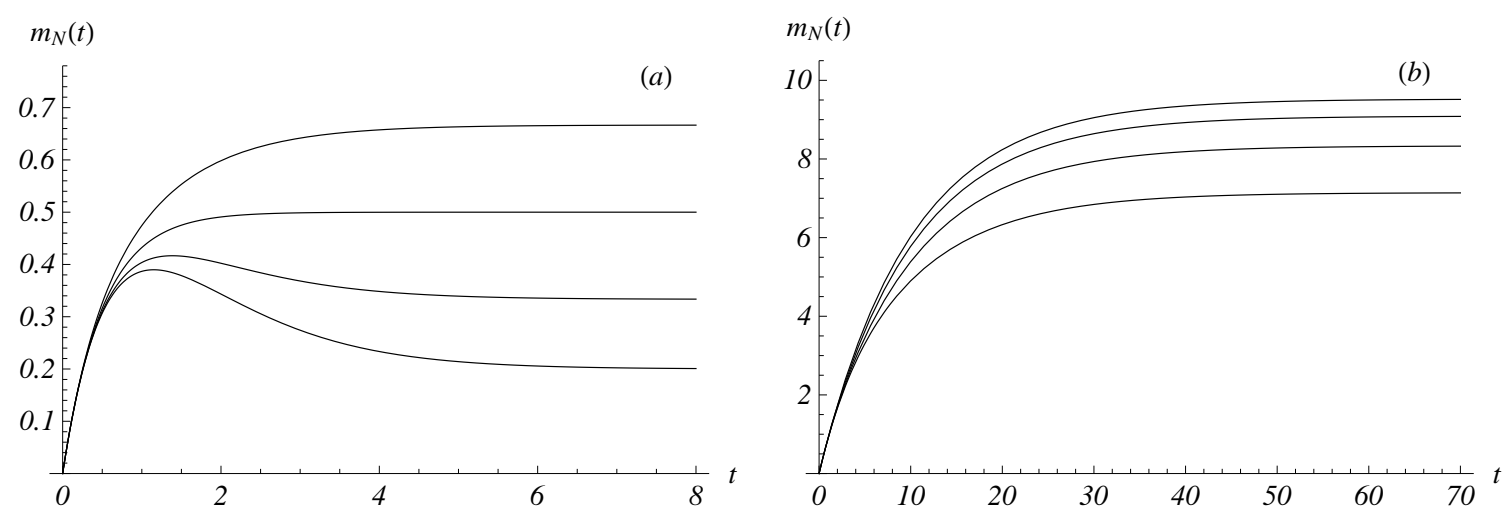

Figure 2: Plots of $m_{N}(t)$ for $\lambda-\mu=1, \eta=0.25,0.5,1,2$ (from bottom to top), and (a) $\nu=1$, (b) $\nu=0.1$. 

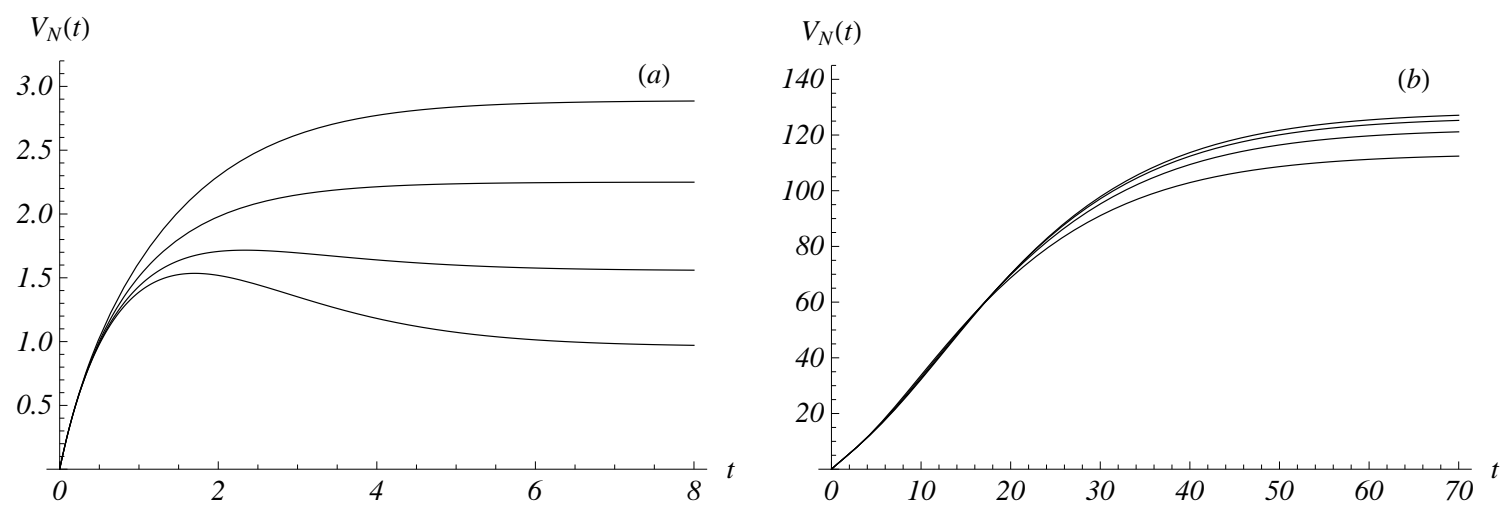

Figure 3: Plots of $V_{N}(t)$ for the same parameters of Figure 2, with $\lambda=2$.

For $t \geq 0$ the transient behaviour of $N_{\varepsilon}^{a}(t)$ is described by the following probabilities:

$$
P_{n}^{a}(t)=P\left\{N_{\varepsilon}^{a}(t)=n \varepsilon\right\}, \quad n \in \mathbb{Z} .
$$

As before, assuming that $P\left\{N_{\varepsilon}^{a}(0)=0\right\}=1$, we have $P_{0}^{a}(0)=1$. Moreover, we note that

$$
\sum_{n \in \mathbb{Z}} P_{n}^{a}(t)=1-q(t)
$$

where $q(t)$ is the probability that $N_{\varepsilon}^{a}(t)$ is in state $F$, and is given in (2.8).

Let us now consider a continuous-time Markov process $\{X(t) ; t \geq 0\}$ having state space $\{F\} \cup(-\infty, \infty)$. This is a jump-diffusion process that describes the motion of a particle that starts from the origin at time 0 , then behaves as a Wiener process with drift $\hat{\lambda}-\hat{\mu}$ and infinitesimal variance $\sigma^{2}$, until it jumps to state $F$ with rate $\nu$. Subsequently it jumps again to the initial state 0 , with rate $\eta$, and diffuses afresh. The probability law of $X(t)$ possesses an absolutely continuous component

$$
f(x, t \mid 0)=\lim _{h \downarrow 0} \frac{1}{h} P\{x \leq X(t)<x+h \mid X(0)=0\}, \quad x \in \mathbb{R}, \quad t \geq 0
$$

and a discrete component $P\{X(t)=F \mid X(0)=0\}$. The latter is easily seen to be identical to the probability $q(t)$, obtained in (2.8).

The continuous approximation relies on the convergence of $N_{\varepsilon}^{a}(t)$ to process $X(t)$ as $\varepsilon$ tends to 0. Probabilities (3.2) are near to $f(n \varepsilon, t \mid 0) \varepsilon$ when $\varepsilon$ is close to zero, and the correspondence between such quantities becomes increasingly better as $\varepsilon$ approaches zero. We thus follow a customary procedure based on the substitution of $P_{n}^{a}(t)$ with $f(n \varepsilon, t \mid 0) \varepsilon$ in the ChapmanKolmogorov forward differential-difference equations of $N_{\varepsilon}^{a}(t)$. After setting $x=n \varepsilon$ and expanding $f$ as Taylor series, we pass to the limit as $\varepsilon \downarrow 0$. By taking positions (3.1) into account and assuming that $\Delta t$ is proportional to $\varepsilon^{2}$, after some calculations we obtain:

$$
\frac{\partial}{\partial t} f(x, t \mid 0)=-\nu f(x, t \mid 0)-(\widehat{\lambda}-\widehat{\mu}) \frac{\partial}{\partial x} f(x, t \mid 0)+\frac{\sigma^{2}}{2} \frac{\partial^{2}}{\partial x^{2}} f(x, t \mid 0), \quad x \neq 0,
$$

to be solved with condition

$$
\int_{-\infty}^{\infty} f(x, t \mid 0) \mathrm{d} x=1-q(t), \quad t \geq 0
$$


Equation (3.3) is the Fokker-Planck equation for a Wiener process perturbed by a leaky term $-\nu f(x, t \mid 0)$. Note that the initial condition $P_{0}^{a}(0)=1$ now becomes

$$
\lim _{t \downarrow 0} f(x, t \mid 0)=\delta(x),
$$

where $\delta(x)$ is the Dirac delta-function.

The above sketched procedure indicates that the process $N_{\varepsilon}^{a}(t)$ converges in some sense to the process $X(t)$ as $\varepsilon$ tends to 0 . Hence, statistical indexes of $X(t)$ are suitable to determine quantities useful to describe $N(t)$ when $\varepsilon$ is very small. Indeed, under position (3.1) we have

$$
P\{N(t)<n \mid N(0)=0\} \simeq P\{X(t)<n \varepsilon \mid X(0)=0\}
$$

recalling that $x=n \varepsilon$, this approximation is expected to improve as $\varepsilon$ goes to zero and as $|n|$ grows larger.

\subsection{Transient and steady-state distributions}

In order to analyse the process $X(t)$ hereafter we obtain the function $f^{*}(x, z \mid 0)$, that is the Laplace transform of density $f(x, t \mid 0)$. Recalling (3.5), from (3.3) we then have

$$
(z+\nu) f^{*}(x, z \mid 0)=-(\widehat{\lambda}-\widehat{\mu}) \frac{\partial}{\partial x} f^{*}(x, z \mid 0)+\frac{\sigma^{2}}{2} \frac{\partial^{2}}{\partial x^{2}} f^{*}(x, z \mid 0), \quad x \neq 0,
$$

whereas condition (3.4) becomes

$$
\int_{-\infty}^{\infty} f^{*}(x, z \mid 0) \mathrm{d} x=\frac{z+\eta}{z(z+\eta+\nu)}
$$

Theorem 3.1 For $z>0$ we have

$$
\begin{aligned}
f^{*}(x, z \mid 0) & =\frac{1}{\sqrt{(\widehat{\lambda}-\widehat{\mu})^{2}+2 \sigma^{2}(z+\nu)}} \frac{(z+\nu)(z+\eta)}{z(z+\eta+\nu)} \\
& \times \exp \left\{\frac{\widehat{\lambda}-\widehat{\mu}}{\sigma^{2}} x-\frac{\sqrt{(\widehat{\lambda}-\widehat{\mu})^{2}+2 \sigma^{2}(z+\nu)}}{\sigma^{2}}|x|\right\} \quad(x \in \mathbb{R}) .
\end{aligned}
$$

Proof. By continuity of $f^{*}(x, z \mid 0)$ in $x=0$ the solution of (3.6) takes the form

$$
f^{*}(x, z \mid 0)= \begin{cases}A e^{w_{2} x} & \text { if } x>0 \\ A e^{w_{1} x} & \text { if } x<0\end{cases}
$$

where

$$
w_{1}, w_{2}=\frac{\widehat{\lambda}-\widehat{\mu} \pm \sqrt{(\widehat{\lambda}-\widehat{\mu})^{2}+2 \sigma^{2}(z+\nu)}}{\sigma^{2}} \quad\left(w_{2}<0<w_{1}\right)
$$

are solutions of equation

$$
\sigma^{2} w^{2}-2(\widehat{\lambda}-\widehat{\mu}) w-2(z+\nu)=0
$$


Making use of (3.9) in (3.7) we thus obtain

$$
A=\frac{1}{\sqrt{(\widehat{\lambda}-\widehat{\mu})^{2}+2 \sigma^{2}(z+\nu)}} \frac{(z+\nu)(z+\eta)}{z(z+\eta+\nu)} .
$$

Hence, Eq. (3.8) finally follows from (3.9), (3.10) and (3.11).

We are now able to obtain an expression of $f(x, t \mid 0)$, that is the analogous of (2.7) for the discrete system.

Theorem 3.2 For all $x \in \mathbb{R}$ and $t \geq 0$ we have

$$
f(x, t \mid 0)=e^{-\nu t} \widetilde{f}(x, t \mid 0)+\eta \int_{0}^{t} q(\tau) e^{-\nu(t-\tau)} \widetilde{f}(x, t-\tau \mid 0) \mathrm{d} \tau,
$$

where $\widetilde{f}(x, t \mid 0)$ is the transition density of a Wiener process $\{\widetilde{X}(t) ; t \geq 0\}$ having drift $\widehat{\lambda}-\widehat{\mu}$ and infinitesimal variance $\sigma^{2}$.

Proof. First of all we note that Eq. (3.8) can be expressed as

$$
\begin{aligned}
f^{*}(x, z \mid 0) & =\left(1+\frac{\eta \nu}{\eta+\nu} \frac{1}{z}-\frac{\eta \nu}{\eta+\nu} \frac{1}{z+\eta+\nu}\right) \exp \left\{\frac{\widehat{\lambda}-\widehat{\mu}}{\sigma^{2}} x\right\} \\
& \times \frac{1}{\sqrt{(\widehat{\lambda}-\widehat{\mu})^{2}+2 \sigma^{2}(z+\nu)}} \exp \left\{-\frac{\sqrt{(\widehat{\lambda}-\widehat{\mu})^{2}+2 \sigma^{2}(z+\nu)}}{\sigma^{2}}|x|\right\} \quad(x \in \mathbb{R}) .
\end{aligned}
$$

Let us now consider a Wiener process having drift $\sqrt{(\widehat{\lambda}-\widehat{\mu})^{2}+2 \sigma^{2} \nu}$ and infinitesimal variance $\sigma^{2}$; its transition density $f_{W}(x, t \mid 0)$ has Laplace transform

$f_{W}^{*}(x, z \mid 0)=\frac{1}{\sqrt{(\widehat{\lambda}-\widehat{\mu})^{2}+2 \sigma^{2}(z+\nu)}} \exp \left\{\frac{\sqrt{(\widehat{\lambda}-\widehat{\mu})^{2}+2 \sigma^{2} \nu}}{\sigma^{2}} x-\frac{\sqrt{(\widehat{\lambda}-\widehat{\mu})^{2}+2 \sigma^{2}(z+\nu)}}{\sigma^{2}}|x|\right\}$,

so that

$$
\frac{\exp \left\{-\frac{|x|}{\sigma^{2}} \sqrt{(\widehat{\lambda}-\widehat{\mu})^{2}+2 \sigma^{2}(z+\nu)}\right\}}{\sqrt{(\widehat{\lambda}-\widehat{\mu})^{2}+2 \sigma^{2}(z+\nu)}}=\exp \left\{-\frac{\sqrt{(\widehat{\lambda}-\widehat{\mu})^{2}+2 \sigma^{2} \nu}}{\sigma^{2}} x\right\} f_{W}^{*}(x, z \mid 0)
$$

Making use of Eq. (3.14), thus Eq. (3.13) yields

$$
f^{*}(x, z \mid 0)=e^{z_{2} x}\left(1+\frac{\eta \nu}{\eta+\nu} \frac{1}{z}-\frac{\eta \nu}{\eta+\nu} \frac{1}{z+\eta+\nu}\right) f_{W}^{*}(x, z \mid 0) \quad(x \in \mathbb{R}),
$$

where we have set

$$
z_{2}=\frac{\widehat{\lambda}-\widehat{\mu}-\sqrt{(\widehat{\lambda}-\widehat{\mu})^{2}+2 \sigma^{2} \nu}}{\sigma^{2}} .
$$

Hence, by taking the inverse Laplace transform in (3.15), we come to

$$
f(x, t \mid 0)=e^{z_{2} x}\left\{f_{W}(x, t \mid 0)+\frac{\eta \nu}{\eta+\nu} \int_{0}^{t}\left[1-e^{-(\eta+\nu)(t-\tau)}\right] f_{W}(x, \tau \mid 0) \mathrm{d} \tau\right\} .
$$


Finally, recalling (2.8) and making use of identity

$$
e^{z_{2} x} f_{W}(x, t \mid 0)=e^{-\nu t} \tilde{f}(x, t \mid 0)
$$

Eq. (3.12) follows.

Some plots of density $f(x, t \mid 0)$ obtained from (3.12) are given in Figure 4.

Hereafter we obtain another expression of $f(x, t \mid 0)$ which is the analogous of (2.11) under the continuous approximation. It involves the first-passage-time density of the Wiener process $\widetilde{X}(t)$ with drift $\widehat{\lambda}-\widehat{\mu}$ and infinitesimal variance $\sigma^{2}$, given by:

$$
\widetilde{g}\left(x, t \mid x_{0}\right)=\frac{\left|x-x_{0}\right|}{t} \widetilde{f}\left(x, t \mid x_{0}\right), \quad x \neq x_{0}, \quad t>0 .
$$

Theorem 3.3 For all $x \in \mathbb{R} \backslash\{0\}$ and $t>0$ we have

$$
f(x, t \mid 0)=\int_{0}^{t} f(0, \tau \mid 0) e^{-\nu(t-\tau)} \widetilde{g}(x, t-\tau \mid 0) \mathrm{d} \tau .
$$

Proof. We recall that for all $x \in \mathbb{R}$ and $t \geq 0$ the following relation holds:

$$
\widetilde{f}(x, t \mid 0)=\exp \left\{\frac{2(\widehat{\lambda}-\widehat{\mu})}{\sigma^{2}} x\right\} \tilde{f}(0, t \mid x) .
$$

Hence, for $x \in \mathbb{R}$ and $t \geq 0$ Eq. (3.12) becomes

$$
f(x, t \mid 0)=e^{-\nu t} \widetilde{f}(x, t \mid 0)+\eta \int_{0}^{t} q(\tau) e^{-\nu(t-\tau)} \exp \left\{\frac{2(\widehat{\lambda}-\widehat{\mu})}{\sigma^{2}} x\right\} \widetilde{f}(0, t-\tau \mid x) \mathrm{d} \tau .
$$

We recall that for the Wiener process $\tilde{X}(t)$ one has:

$$
\widetilde{f}(0, t \mid x)=\int_{0}^{t} \widetilde{g}(0, \tau \mid x) \widetilde{f}(0, t-\tau \mid 0) \mathrm{d} \tau, \quad x \neq 0, \quad t>0 .
$$

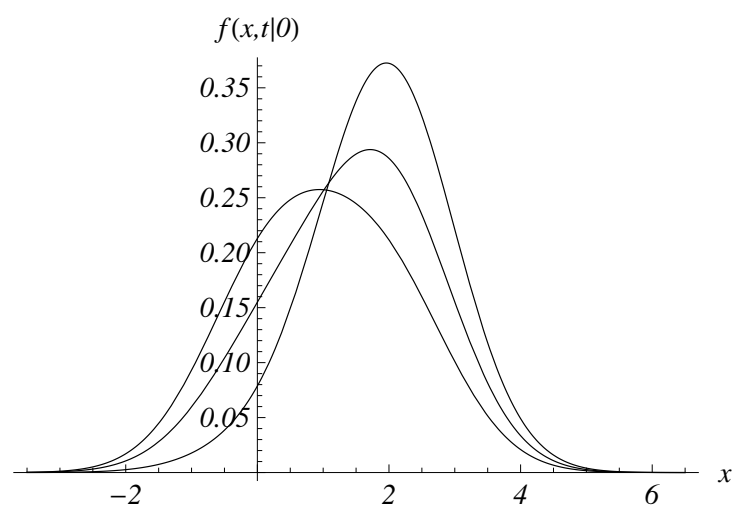

Figure 4: Plots of density (3.12), for $\widehat{\lambda}-\widehat{\mu}=2, \eta=1, \sigma^{2}=1, t=1$, and $\nu=1,0.5,0.1$ (from left to right). The probability mass is respectively $0.5677,0.7410,0.9394$, due to (3.4). 
Moreover, making use of (3.16) and (3.17), for $x \neq 0$ and $t>0$ we have

$$
\begin{aligned}
\widetilde{g}(0, t \mid x) & =\frac{|x|}{t} \exp \left\{-\frac{2(\widehat{\lambda}-\widehat{\mu})}{\sigma^{2}} x\right\} \widetilde{f}(x, t \mid 0) \\
& =\exp \left\{-\frac{2(\widehat{\lambda}-\widehat{\mu})}{\sigma^{2}} x\right\} \widetilde{g}(x, t \mid 0) .
\end{aligned}
$$

Substituting (3.19) in the last term of (3.18), employing Fubini's theorem and making use again of (3.12) we finally obtain:

$$
\begin{aligned}
f(x, t \mid 0)= & e^{-\nu t} \widetilde{f}(x, t \mid 0)-e^{-\nu t} \exp \left\{\frac{2(\widehat{\lambda}-\widehat{\mu})}{\sigma^{2}} x\right\} \int_{0}^{t} \widetilde{g}(0, u \mid x) \widetilde{f}(0, t-u \mid 0) \mathrm{d} u \\
& +\exp \left\{\frac{2(\widehat{\lambda}-\widehat{\mu})}{\sigma^{2}} x\right\} \int_{0}^{t} \widetilde{g}(0, u \mid x) e^{-\nu u} f(0, t-u \mid 0) \mathrm{d} u, \quad x \neq 0 .
\end{aligned}
$$

The first and the second term in the right-hand-side of (3.21) vanish due to Eqs. (3.17) and (3.19). Hence, the thesis follows making use of (3.20) in the last term of (3.21).

Aiming to analyse the asymptotic behaviour of process $X(t)$ we now introduce the steadystate density

$$
W(x)=\lim _{t \rightarrow+\infty} f(x, t \mid 0), \quad x \in \mathbb{R} .
$$

Hereafter we obtain that $W(x)$ is a bilateral asimmetric exponential density.

Theorem 3.4 The steady-state density (3.22) is given by

$$
W(x)=\frac{\eta \nu}{\eta+\nu} \frac{1}{\sqrt{(\widehat{\lambda}-\widehat{\mu})^{2}+2 \sigma^{2} \nu}} \exp \left\{\frac{\widehat{\lambda}-\widehat{\mu}}{\sigma^{2}} x-\frac{\sqrt{(\widehat{\lambda}-\widehat{\mu})^{2}+2 \sigma^{2} \nu}}{\sigma^{2}}|x|\right\}, \quad x \in \mathbb{R} .
$$

Proof. Being $W(x)=\lim _{z \downarrow 0}\left[z f^{*}(x, z \mid 0)\right]$, from (3.8) we have (3.23).

We remark that, due to (3.4) and (2.14), we have

$$
\int_{-\infty}^{\infty} W(x) \mathrm{d} x=1-q=\frac{\eta}{\eta+\nu}
$$

\subsection{Mean and variance}

Let us now define the mean

$$
m_{X}(t):=E\left[X(t) \cdot \mathbf{1}_{\{X(t) \neq F\}} \mid X(0)=0\right], \quad t \geq 0 .
$$

Due to (3.12) and recalling that $E[\tilde{X}(t) \mid \tilde{X}(0)=0]=(\widehat{\lambda}-\widehat{\mu}) t$, for all $t \geq 0$ we have

$$
m_{X}(t)=e^{-\nu t}(\widehat{\lambda}-\widehat{\mu}) t+\eta \int_{0}^{t} q(\tau) e^{-\nu(t-\tau)}(\widehat{\lambda}-\widehat{\mu})(t-\tau) \mathrm{d} \tau
$$



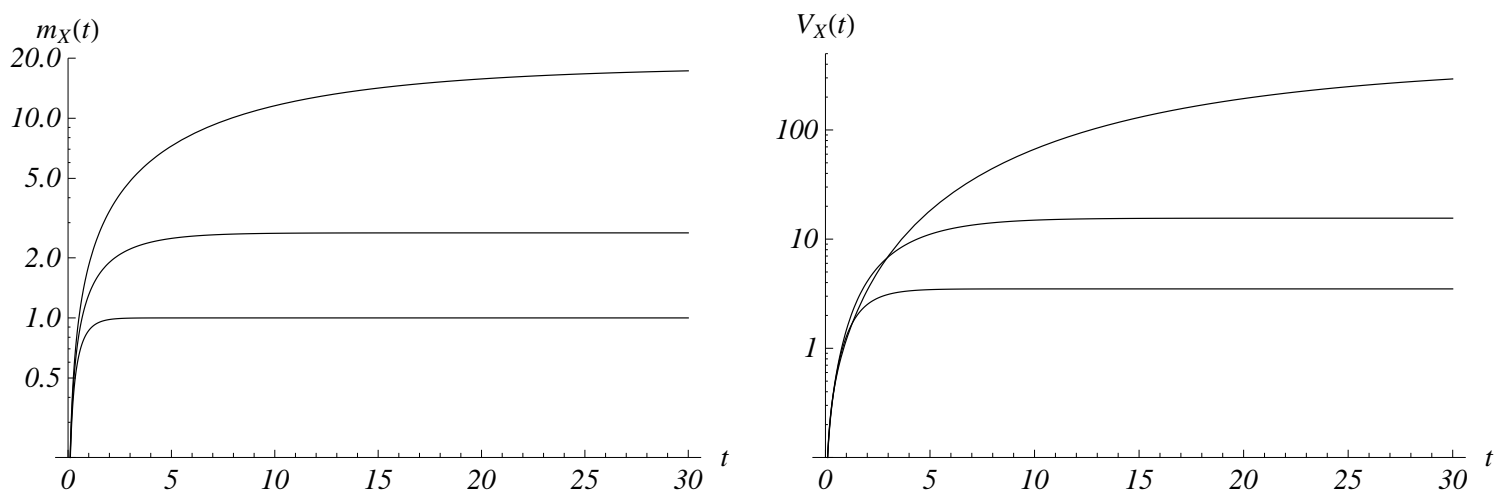

Figure 5: Plots of mean and variance (3.24) and (3.25) for the same values of the parameters as in Figure 4 (from bottom to top).

Use of (2.8) and straightforward calculations thus yield:

$$
m_{X}(t)=\frac{(\widehat{\lambda}-\widehat{\mu}) \eta}{(\eta+\nu) \nu}\left\{1-e^{-\nu t}+\frac{\nu^{2}}{\eta^{2}} e^{-\nu t}\left(1-e^{-\eta t}\right)\right\}, \quad t \geq 0
$$

The strict correspondence between the means (2.18) and (3.24) is evident.

Making use of (3.12) one can express the moments of $X(t)$ in terms of the moments of the Wiener process. In particular, for any $t \geq 0$ we have:

$$
\begin{aligned}
V_{X}(t) & :=\operatorname{Var}\left[X(t) \cdot \mathbf{1}_{\{X(t) \neq F\}} \mid X(0)=0\right] \\
& =\frac{\sigma^{2} \eta}{(\eta+\nu) \nu}\left[1-e^{-\nu t}+\frac{\nu^{2}}{\eta^{2}} e^{-\nu t}\left(1-e^{-\eta t}\right)\right] \\
& +\frac{(\widehat{\lambda}-\widehat{\mu})^{2}}{(\eta+\nu)^{2} \nu^{2} \eta^{2}}\left\{-2 \nu^{2} e^{-\nu t}\left(1-e^{-\eta t}\right)\left(\nu^{2}+\eta \nu+\eta^{2}\right)+2 \nu \eta^{3}\left(1-e^{-\nu t}\right)\right. \\
& \left.+\eta^{4}+2 \eta \nu(\eta+\nu)\left(\nu^{2}-\eta^{2}\right) t e^{-\nu t}-e^{-2 \nu t}\left(\nu^{2}-\eta^{2}-\nu^{2} e^{-\eta t}\right)^{2}\right\} .
\end{aligned}
$$

Notice that for the densities shown (from left to right) in Figure 4 we have $m_{X}(1)=0.865,1.305$, 1.834 and $V_{X}(1)=1.283,1.469,1.198$ (remarking that in this case $V_{X}(1)$ is not monotonic in $\nu)$. The behaviour of mean and variance as $t$ varies is shown in Figure 5.

Finally, from (3.24) and (3.25) we immediately obtain the asymptotic limits of the mean and variance of the approximating process:

$$
\lim _{t \rightarrow+\infty} m_{X}(t)=\frac{(\widehat{\lambda}-\widehat{\mu}) \eta}{(\eta+\nu) \nu}, \quad \lim _{t \rightarrow+\infty} V_{X}(t)=\frac{\sigma^{2} \eta}{(\eta+\nu) \nu}+\frac{(\widehat{\lambda}-\widehat{\mu})^{2} \eta}{(\eta+\nu)^{2} \nu^{2}}(2 \nu+\eta) .
$$

\section{Remarks on the continuous approximation}

We conclude the paper by giving some comments concerning the goodness of the continuous approximation. Recall that, as pointed out in Section 3, the approximation is expected to improve as $\varepsilon$ goes to zero and as $|n|$ grows larger. 
First of all we point out that the means of the discrete process and of the continuous approximating process are in strict agreement. Indeed, by comparing (2.18) and (3.24), under substitutions (3.1) we immediately have

$$
E[N(t) \mid N(0)=0]=E\left[\frac{X(t)}{\varepsilon} \mid \frac{X(0)}{\varepsilon}=0\right]
$$

for all $\varepsilon>0$ and $t \geq 0$.

In order to ascertain the approximation between the transient distributions of $N(t)$ and $X(t)$, let us now denote by $P_{n}^{*}(z ; \varepsilon), n \in \mathbb{Z}$, the Laplace transform of $P_{n}(t)$ where the substitutions (3.1) are performed. Hence, making use of (2.15) and (3.8) we obtain

$$
\lim _{\varepsilon \downarrow 0} \frac{P_{0}^{*}(z ; \varepsilon)}{\varepsilon}=\frac{\eta \nu}{z(z+\eta+\nu) \sqrt{(\widehat{\lambda}-\widehat{\mu})^{2}+2(z+\nu) \sigma^{2}}}+\frac{1}{\sqrt{(\widehat{\lambda}-\widehat{\mu})^{2}+2(z+\nu) \sigma^{2}}}=f^{*}(0, z \mid 0) .
$$

Moreover, by noting that under positions (3.1)

$$
\lim _{\varepsilon \downarrow 0} \frac{z+\lambda+\mu+\nu-\sqrt{(z+\lambda+\mu+\nu)^{2}-4 \lambda \mu}}{2 \mu}=1,
$$

from (2.13) for $x \neq 0$ we have

$$
\lim _{\varepsilon \downarrow 0} \frac{\left.P_{n}^{*}(z ; \varepsilon)\right|_{n=x / \varepsilon}}{\varepsilon}=\left\{\begin{array}{ll}
e^{w_{2} x} f^{*}(0, z \mid 0), & x>0 \\
e^{w_{1} x} f^{*}(0, z \mid 0), & x<0
\end{array}\right\}=f^{*}(x, z \mid 0),
$$

where $f^{*}(x, z \mid 0)$ is given in (3.8) and where $w_{1}$ and $w_{2}$ are defined in (3.10). Hence, recalling the meaning of $P_{n}^{*}(z ; \varepsilon)$ and denoting by $P_{n}(t ; \varepsilon)$ the probabilities $(2.9)$ when the substitutions (3.1) are performed, we have:

$$
\lim _{\varepsilon \downarrow 0} \frac{\left.P_{n}(t ; \varepsilon)\right|_{n=x / \varepsilon}}{\varepsilon}=f(x, t \mid 0) .
$$

This implies that under the approximating procedure the state probabilities of the discrete process are close to the density $f(x, t \mid 0)$ of process $X(t)$. In addition, this is confirmed by the data of Table 1 showing that the agreement of the two steady-state distributions improves as $\varepsilon$ decreases and as $|n|$ increases. Furthermore, we remark that performing the substitutions

$$
\varepsilon_{h}=\varepsilon h, \quad \widehat{\lambda}_{h}=\widehat{\lambda} h, \quad \widehat{\mu}_{h}=\widehat{\mu} h, \quad \sigma_{h}^{2}=\sigma^{2} h, \quad(h>0)
$$

recalling (3.1) we have

$$
\lambda_{h}:=\frac{\widehat{\lambda}_{h}}{\varepsilon_{h}}+\frac{\sigma_{h}^{2}}{2 \varepsilon_{h}^{2}}=\lambda, \quad \mu_{h}:=\frac{\widehat{\mu}_{h}}{\varepsilon_{h}}+\frac{\sigma_{h}^{2}}{2 \varepsilon_{h}^{2}}=\mu .
$$

Hence, carrying out substitutions (4.1) in the steady-state density (3.23) we obtain $W_{h}\left(n \varepsilon_{h}\right) \varepsilon_{h}=$ $W(n \varepsilon) \varepsilon$ for any $h>0$. This shows that the approximation procedure is effective when the rates $\lambda$ and $\mu$ are suitably varying.

In conclusion we point out that the agreement between the discrete model and the approximating jump-diffusion process is also confirmed by the comparison of their asymptotic means and variances. Indeed, the correspondence of limits (3.26) with those obtained in Eqs. (2.19) and (2.20) for the discrete model is immediate under positions (3.1). 


\begin{tabular}{|r|ccc|ccc|ccc|}
\hline & \multicolumn{3}{|c|}{$\varepsilon=0.1$} & \multicolumn{3}{|c|}{$\varepsilon=0.05$} & \multicolumn{3}{c|}{$\varepsilon=0.01$} \\
\hline$n$ & $\pi_{n}(\varepsilon) / \varepsilon$ & $W(n \varepsilon)$ & $\Delta(n)$ & $\pi_{n}(\varepsilon) / \varepsilon$ & $W(n \varepsilon)$ & $\Delta(n)$ & $\pi_{n}(\varepsilon) / \varepsilon$ & $W(n \varepsilon)$ & $\Delta(n)$ \\
\hline-6 & 0.03621 & 0.03668 & 0.01305 & 0.04073 & 0.04102 & 0.00721 & 0.04480 & 0.04487 & 0.00155 \\
-5 & 0.03757 & 0.03807 & 0.01353 & 0.04149 & 0.04180 & 0.00733 & 0.04496 & 0.04503 & 0.00156 \\
-4 & 0.03897 & 0.03952 & 0.01401 & 0.04227 & 0.04258 & 0.00745 & 0.04513 & 0.04520 & 0.00156 \\
-3 & 0.04044 & 0.04102 & 0.01448 & 0.04306 & 0.04339 & 0.00757 & 0.04530 & 0.04537 & 0.00157 \\
-2 & 0.04196 & 0.04258 & 0.01497 & 0.04387 & 0.04420 & 0.00769 & 0.04547 & 0.04554 & 0.00157 \\
-1 & 0.04353 & 0.04420 & 0.01544 & 0.04469 & 0.04503 & 0.00781 & 0.04564 & 0.04571 & 0.00158 \\
0 & 0.04516 & 0.04588 & 0.01593 & 0.04552 & 0.04588 & 0.00793 & 0.04581 & 0.04588 & 0.00158 \\
1 & 0.04260 & 0.04323 & 0.01472 & 0.04420 & 0.04454 & 0.00763 & 0.04554 & 0.04561 & 0.00157 \\
2 & 0.04019 & 0.04073 & 0.01351 & 0.04292 & 0.04323 & 0.00732 & 0.04527 & 0.04534 & 0.00156 \\
3 & 0.03791 & 0.03838 & 0.01231 & 0.04167 & 0.04196 & 0.00702 & 0.04500 & 0.04507 & 0.00154 \\
4 & 0.03576 & 0.03616 & 0.01111 & 0.04046 & 0.04073 & 0.00672 & 0.04473 & 0.04480 & 0.00153 \\
5 & 0.03373 & 0.03407 & 0.00990 & 0.03929 & 0.03954 & 0.00641 & 0.04447 & 0.04454 & 0.00152 \\
6 & 0.03182 & 0.03210 & 0.00870 & 0.03814 & 0.03838 & 0.00611 & 0.04421 & 0.04427 & 0.00151 \\
\hline
\end{tabular}

Table 1: For $\eta=0.25, \nu=1, \widehat{\lambda}=1, \widehat{\mu}=2$ and $\sigma=3$ the quantities $\pi_{n}(\varepsilon) / \varepsilon$ and $W(n \varepsilon)$ are listed together with the relative difference $\Delta(n):=\left[W(n \varepsilon) \varepsilon-\pi_{n}(\varepsilon)\right] / \pi_{n}(\varepsilon)$ for various integer values of $n$ and various choices of $\varepsilon$. We have $(\lambda, \mu)=(460,470)$ when $\varepsilon=0.1,(\lambda, \mu)=(1820,1840)$ when $\varepsilon=0.05$, and $(\lambda, \mu)=(45100,45200)$ when $\varepsilon=0.01$.

\section{Acknowledgments}

The constructive criticism of two anonymous reviewers is gratefully acknowledged.

This work has been partially performed during a visit of Dr B. Krishna Kumar at Salerno University. He would like to acknowledge the Department of Mathematics and Informatics for kindness and hospitality. The support by MIUR (PRIN 2008) and by GNCS-INdAM is acknowledged by the other authors.

\section{References}

[1] Abate J, Kijima M, Whitt W (1991) Decomposition of the $M / M / 1$ transition function. Queueing Systems 9:323-336

[2] Baccelli F, Massey WA (1989) A sample path analysis of the $M / M / 1$ queue. J Appl Prob 26:418-422

[3] Brockwell PJ (1985) The extinction time of a birth, death and catastrophe process and of a related diffusion model. Adv Appl Prob 17:42-52

[4] Buonocore A, Di Crescenzo A, Giorno V, Nobile AG, Ricciardi LM (2009) A Markov chain-based model for actomyosin dynamics. Scientiae Math Japon 70:159-174

[5] Chao X, Zheng Y (2003) Transient analysis of immigration-birth-death process with total catastrophes. Prob Eng Inform Sci 17:83-106

[6] Conolly BW (1971) On randomized random walks. SIAM Review 13:81-99

[7] Conolly BW, Parthasarathy PR, Selvaraju N (2002) Doubled-ended queues with impatience. Computers Oper Res 29:2053-2072 
[8] Di Crescenzo A, Giorno V, Nobile AG, Ricciardi LM (2003) On the $M / M / 1$ queue with catastrophes and its continuous approximation. Queueing Systems 43:329-347

[9] Di Crescenzo A, Giorno V, Nobile AG, Ricciardi LM (2008) A note on birth-death processes with effective catastrophes. Stat Prob Lett 78:2248-2257

[10] Di Crescenzo A, Nastro A (2004) On first-passage-time densities for certain symmetric Markov chains. Scientiae Math Japon 60:381-390

[11] Di Crescenzo A, Nobile AG (1995) Diffusion approximation to a queueing system with timedependent arrival and service rates. Queueing Systems 19:41-62

[12] Economou A, Fakinos D (2003) A continuous-time Markov chain under the influence of a regulating point process and applications in stochastic models with catastrophes. Europ J Operat Res 149:625640

[13] Economou A, Fakinos D (2008) Alternative approaches for the transient analysis of Markov chains with catastrophes. J Statist Theory Pract 2:183-197

[14] Gani J, Swift RJ (2006) A simple approach to birth processes with random catastrophes. J Combin Inform System Sci 31:1-7

[15] Gani J, Swift RJ (2007) Death and birth-death and immigration processes with catastrophes. J Statist Theory Pract 1:39-48

[16] Giorno V, Nobile AG, Ricciardi LM (1986) On some diffusion approximations to queueing systems. Adv Appl Prob 18:991-1014

[17] Giorno V, Nobile AG, Ricciardi LM (1987) On some time non-homogeneous diffusion approximations to queueing systems. Adv Appl Prob 19:974-994

[18] Jain JL, Mohanty SG, Boehm W (2007) A Course on Queueing Models. Chapman and Hall/CRC, Boca-Raton

[19] Kella O, Whitt W (1990) Diffusion approximation for queues with server vacations. Adv Appl Prob 22:706-729

[20] Kimura T (2004) Diffusion models for computer/communication systems. Econ J Hokkaido Univ $33: 37-52$

[21] Krinik A, Mortensen C (2007) Transient probability functions of finite birth-death processes with catastrophes. J Statist Plann Infer 137:1530-1543

[22] Krinik A, Rubino G, Marcus D, Swift R, Kasfy H, Lam H (2005) Dual processes to solve single server systems. J Statist Plann Infer 135:121-147

[23] Krishna Kumar B, Arivudainambi D (2000) Transient solution of an $M / M / 1$ queue with catastrophes. Comput Math Appl 40:1233-1240

[24] Krishna Kumar B, Pavai Madheswari S (2003) Transient solution of an $M / M / 2$ queue with catastrophes. Math Scientist 28:129-136

[25] Krishna Kumar B, Krishnamoorthy A, Pavai Madheswari S, Sadiq Basha S (2007) Transient analysis of a single server queue with catastrophes, failures and repairs. Queueing Syst 56:133-141

[26] Parthasarathy PR, Lenin RB (2004) Birth and death processes (BDP) models with applicationsqueueing communication systems, chemical models. Biological models: the state-of-the-art with a time-dependent perspective. Amer J Math Management Sci 24:1-212 
[27] Stirzaker D (2006) Processes with catastrophes. Math Scientist 31:107-118

[28] Stirzaker D (2007) Processes with random regulation. Prob Eng Inform Sci 21:1-17

[29] Swift RJ (2000) A simple immigration-catastrophe process. Math Scientist 25:32-36

[30] Swift RJ (2001) Transient probabilities for a simple birth-death-immigration process under the influence of total catastrophes. Int J Math Math Sci 25:689-692

[31] Switkes J (2004) An unbiased random walk with catastrophe. Math Scientist 29:115-121 